\title{
La modernización en Chile: sociedad civil compulsiva o congestionada ¿es factible el cambio social?
}

\section{Pablo Rivera Vargas*}

\section{RESUMEN}

Los cambios sociales experimentados por Chile, están marcados por el proceso de modernización adoptada por la clase dirigencial para propiciar un determinado modelo de modernización. Este hecho ha tenido múltiples consecuencias en las distintas estructuras sociales, dado el sentido capitalista que el modelo promueve, donde los resultados no son equitativos en términos de repartición de excedentes, pero si a nivel de aceptación y asimilación social.

Palabras clave: Modernidad / Modernización / Compulsión social / Congestión y malestares / subpolitización.

\section{The modernization in Chile: compulsive or congested civil society. Is the social change feasible?}

\section{ABSTRACT}

The social changes experienced in Chile have been marked by the process of modernization adopted by the leading class to cause a certain model of modernization. This fact has had multiple consequences in the different social structures, given the capitalist sense promoted by that model, where the results are not equitable in terms of distribution of surpluses, but concerning acceptance and social assimilation.

Key words: Modern age / modernization / social compulsion / congestion and discomfort / subpolitization

* Chileno. Sociólogo. Académico Departamento de Trabajo Social, Universidad Cardenal Silva Henríquez. Correo electrónico: riveravargaspablo@gmail.com 


\section{Modernidad y modernización}

La búsqueda de la Modernidad, desde su concepción occidental, en los proyectos políticos y económicos de Chile, principalmente en el siglo XX y XXI, es resaltada en diversos ámbitos de análisis, como el hecho más determinante a la hora de reflexionar sobre la construcción de la sociedad chilena.

Morandé (1987) considera que los grandes problemas de la historia de Chile y de gran parte de Latinoamérica, radican en una aplicación incompleta y voluntarista de la modernidad, centrada más que nada en la modernización productiva, que en la adopción de los ideales libertarios e igualitarios de la Revolución Francesa. Para el autor, la aplicación de este tipo de modernidad es algo importado desde afuera por una coyuntura histórica determinada, que consiste en la invasión francesa a España en 1808. Esta es la ruptura radical de la historia americana, pues marca el quiebre con nuestro verdadero ethos mestizo, barroco y católico-ritual. Desde ese punto hasta hoy, todo lo que ha existido es foráneo, impuesto, alienado.

Quijano (1989) considera que la Modernidad en América Latina no es un fenómeno desconectado de la historia de la Modernidad Europea. No solo porque fue resultado de la relación colonial, ni porque es un fenómeno que se impone en la región con "relativa tardía", sino ante todo porque su consolidación y su prolongada duración "que aún no termina del todo" fueron a su vez, asociadas al hecho de que en Europa la dominación pudo imponer, en su propio servicio contra la liberación, una casi completa instrumentalización de la razón. "Desde sus propios inicios, la Ilustración Europea contiene una división que pronto revelará insanable entre las tendencias para las cuales la racionalidad es una genuina promesa de liberación de la humanidad, de sus propios fantasmas; de la sociedad, de las prisiones del poder. Y del otro lado; las tendencias para las cuales la racionalidad es un dispositivo instrumental del poder, de la dominación" (Quijano 1989: 53).

Lo central en esta idea de Quijano es que las primeras tendencias estaban difundidas sobre todo en la Europa Mediterránea, la Europa Latina. En tanto que las segundas, tenían el predominio de la Europa Nórdica, y en especial de lo que es hoy Gran Bretaña. Esa diferenciación se hace más clara y aguda en el curso del siglo XVIII; toma parte en el conflicto de poder entre Inglaterra y España, y después entre Inglaterra y Francia, y fue ciertamente definitiva, con la 
imposición de la hegemonía Inglesa sobre el resto de Europa y en el siglo XIX sobre la mayor parte del mundo.

La imposición de la hegemonía Británica, desde fines del siglo XVIII y durante todo el siglo XIX, significó también la hegemonía de las tendencias que no podían concebir la racionalidad de otro modo que como arsenal instrumental del poder y de la dominación. La asociación entre razón y liberación quedó oscurecida de ese modo. Para Quijano, la modernidad sería vista en adelante a través "del enturbiado espejo de la modernización, es decir; la transformación del mundo, de la Sociedad, según las necesidades de la dominación" (Quijano 1988: 53). Y específicamente de la dominación del capital, despojado de toda otra finalidad que la acumulación.

Para Chile, esa inflexión de la historia fue no sólo decisiva, si no también catastrófica; "La victoria de la instrumentalización de la razón en servicio de la dominación, fue también una profunda derrota para América Latina, pues por su propia situación colonial, la producción de la racionalidad moderna estuvo aquí asociada, sobre todo, a las promesas liberadoras de la modernidad" (Quijano 1988: 54). La metamorfosis de aquella quedaría destinada a durar por un período histórico muy prolongado. Chile no se volvería a encontrar la modernidad, si no bajo la cubierta de la "Modernización".

Según se expresa en el Informe de Desarrollo Humano del PNUD de 1998, La modernidad es entendida como un horizonte normativo y como proyecto. Es la búsqueda de paz social, igualdad y libertad mediante la emancipación de la subjetividad y a través de la liberación de la creatividad de los sistemas sociales. Por su parte, la modernización por el contrario, es el modo histórico mediante el cual se instaura la modernidad en el plano de los sistemas e instituciones sociales. "La modernización actual se caracteriza porque los sistemas sociales se autonomizan y se diferencian cada vez más como efecto de la aplicación sostenida de la racionalidad instrumental a sus procesos, y por la desregulación, esto es, por el debilitamiento de los vínculos normativos que relacionan a los sistemas entre si" (PNUD 1998: 64).

Según esta descripción, podríamos plantear que en la sociedad chilena predomina una racionalización medios-fines propia de la modernización. Es decir, un arsenal instrumental del poder y de la dominación, que construye y legitima una creciente polarización social entre quienes acceden directamente al poder y quienes lo buscan y no lo tienen. 
En un intento de dar consistencia a la reflexión respecto de las transformaciones y cambios sociales vividos por nuestro país en los últimos años, sustentaremos el análisis a partir del posicionamiento de nuestra sociedad en la búsqueda de la Modernidad y de la Modernización, resaltando en ella, fenómenos claves como son la "Compulsividad" e "individualización" que en términos de Giddens (1993), representarían rasgos distintivos de las sociedades que viven un proceso medianamente consolidado de modernización social en términos occidentales capitalistas; y "Subpolitización" que según el mismo autor, y Beck (2000), caracterizaría a las sociedades que presentan cierto nivel de incertidumbre y desencanto hacia la lógica de modernización elegida por la clase política y el resto de los sistemas abstractos (estado, saber científico, nuevas tecnologías), para conseguir la modernidad.

\section{Compulsión social en Chile}

El concepto de sociedad compulsiva se aleja diametralmente de la tradición. Principalmente incide el advenimiento de la modernidad industrial, que trae consigo códigos y símbolos nuevos, distintos a los propios de una cultura específica. Mismo concepto, quizás más ligado a la conformación de los estados es el que utiliza Garretón (2000) en su definición de sociedad de ruptura. En pocas palabras, tradición sin tradicionalismo.

En términos generales, "La compulsión es una confianza congelada, un compromiso que no tiene objeto sino que se auto-perpetúa e impide reanudar el compromiso con los sistemas abstractos que han llegado a dominar el contenido de la vida cotidiana" (Giddens 1993: 88-89).

Este fenómeno también está caracterizado por la emergencia de nuevos actores sociales y de múltiples diversidades de comportamiento, que requieren de una sociedad dispuesta a la tolerancia, ya que de no existir esta, chocaría con los valores tradicionales, lo que estancaría el proceso de modernización en el que Chile está involucrado. En el fondo este fenómeno de individualización de la ciudadanía termina siendo más funcional al sistema de vida presente, que una reacción crítica del mismo.

Sin embargo, no se trata de un fenómeno reciente, ya en el 2000, el informe del PNUD plantea la existencia de cuatro características troncales del proceso de individualización que caracteriza al Chile de la época: 
- Una biografía construida: Cada vez menos los jóvenes chilenos deciden sus planes de vida acorde a las costumbres de antaño o las pautas sociales vigentes. "Ni siquiera optan según las indicaciones de sus padres". La biografía personal es una construcción. "El individuo está definido a partir de ciertas características: su vocación y su condición socioeconómica, sus habilidades, ejemplos de su grupo de referencia inmediata e información de los medios de comunicación masivos" (PNUD 2000: 29). A partir de ello el o la joven explora su porvenir, somete a pruebas sus experiencias laborales, construye un proyecto de vida siempre guiado por un ideal de autorealización individual.

- La construcción de sentidos: La individualización implica que la persona decide, según su conciencia personal, creencias, valores y normas de su conciencia cotidiana, lo que modifica las relaciones interpersonales. "En el diario vivir puede percibirse como las verdades consagradas son sometidas a discusión, las pautas sociales se vuelven más flexibles y son menos los valores compartidos" (PNUD 2000: 29).

- El individuo reflexivo: Según el informe, el individuo moderno ya no puede guiarse por tradiciones y costumbres, ya que en un mundo siempre cambiante como el presente, las rutinas no bastan. "En forma constante el individuo se encuentra enfrentado a tomar decisiones: el empleo más conveniente, el barrio donde vive, la escuela para los hijos, el plan de salud adecuado, el fondo de pensión más rentable, etcétera" (PNUD 2000: 30). En suma, toda persona adulta está obligada a reflexionar a diario sobre su entorno y los cursos de acción posibles.

- La individualización brinda una nueva oportunidad: En la actualidad el/la chileno/a cuenta con la libertad de escoger lo que quiere ser. Es bajo estas condiciones que la persona se transforma en sujeto de derechos y obligaciones y asume la responsabilidad de sí mismo.

Ahora bien, el elogio de la "libertad de elegir" resulta falso cuando silencia las dificultades propias a la individualización. Hay que asumir "las tribulaciones del Yo" (Giddens 1997); esto es un conjunto de dilemas que enfrenta el individuo moderno cuando está obligado a elegir de modo reflexivo su biografía. Tal elección es compulsiva; las personas están obligadas, lo quieran o no, a diseñar, fabricar y escenificar su identidad. Aflora la subjetividad y sus interrogantes 
impregnan todas las relaciones (¿quién soy yo? ¿Qué deseo? ¿Qué debo hacer?).

Sin embargo, se desvanecen los criterios de selección que, por lo general, canalizaban esa búsqueda de sí mismo. "Las oportunidades, amenazas, ambivalencias biográficas que anteriormente era posible superar en un grupo familiar, en la comunidad de aldea o recurriendo a la clase o grupo social, tienen que ser en forma progresiva, percibidas, interpretadas y manejadas por los propios individuos. (...) Ahora se espera de los individuos que sean capaces de dominar esas "oportunidades arriesgadas" sin que puedan, debido a la complejidad de la sociedad moderna, tomar las decisiones necesarias sobre una base bien fundada y responsable; es decir, considerando las posibles consecuencias" (Beck 1997: 21).

\section{La sociedad civil chilena y su vínculo con las estructuras abstractas}

Pareciera ser que la principal herramienta de legitimidad que posee la plataforma de la modernización actual en Chile, es el estrecho vínculo entre la ciudadanía con la mayoría de los sistemas abstractos. Frente a esto podríamos plantear que es el Estado quien aparece como la estructura abstracta más legitimada. Según el informe del PNUD 2004, la mayoría de la población reconoce al Estado como la institución con mayor poder en la sociedad. Sin embargo, es interesante constatar que dentro de este selecto grupo de instituciones que son vistas por la ciudadanía como entes con poder, están también el empresariado y los medios de comunicación, ambos componentes clave de la legitimidad de la actual dinámica modernizadora. Por el contrario, en este mundo globalizado e individualizado, las instituciones que pierden legitimidad y a la vez poder según la opinión de la ciudadanía, serían fundamentalmente los sindicatos y las organizaciones sociales. Sumado a lo anterior, también la clase política partidista es vista como una institución carente de poder, lo que según se describe en el informe del PNUD 2004, alcanza niveles preocupantes. Para Garretón (2005) esto se debería a que el actual diseño político limita mucho el desenvolvimiento de la ciudadanía, ya que se reduce solo a dos opciones que no logran generar representatividad en gran parte de la población, como si lo hicieron en un momento: "Por un lado tenemos una derecha antidemocrática vinculada a una dictadura y que reniega de sí misma, y por otro lado, una concertación que si bien alguna vez representó los intereses de la mayoría de la ciudadanía en la actualidad es vista como el mal menor" (Garretón 2005). 


\section{Saber científico y tecnológico}

En gran medida este tipo de dinámica hacia la modernización, está consolidado en el alma de la sociedad chilena, dada la confianza en el saber tecnológico y científico de nuestra población, expresado permanentemente en el intento de acceder a ellas, sin que su consumo genere aún mayores incertidumbres.

"El vínculo entre la ciudadanía y el saber científico y tecnológico es intenso, consecuencia de la forma en que se instaura el concepto de modernización en Chile" (PNUD 2006). Existe un pensamiento necesariamente positivo hacia el desarrollo tecnológico, el que es según esta idea colectiva, la respuesta necesaria y suficiente a cualquier problemática presente y futura. Este hecho también es ratificado en el informe del PNUD 2006 (ver cuadro 1), donde se muestra que el 68,8\% de la población considera que la tecnología es una herramienta fundamental para el desarrollo del país, y donde el $61,1 \%$ ve en la emergencia de las nuevas tecnologías más oportunidades que amenazas (ver cuadro 2).

Cuadrol.

\begin{tabular}{|l|l|}
\hline \multicolumn{2}{|l|}{$\begin{array}{l}\text { Pensando en Chile, ¿cuál de las siguientes frases representa mejor su } \\
\text { opinión? }\end{array}$} \\
\hline $\begin{array}{l}\text { La tecnología es una herramienta fundamental para que nos } \\
\text { desarrollemos como país. }\end{array}$ & $68,8 \%$ \\
\hline $\begin{array}{l}\text { La tecnología no ayuda a solucionar los problemas más importantes } \\
\text { para el desarrollo del país. }\end{array}$ & $27,2 \%$ \\
\hline No Sabe - No Responde & $4,0 \%$ \\
\hline
\end{tabular}

Fuente: PNUD 2006: 234.

Cuadro 2.

\begin{tabular}{|l|l|}
\hline En general, pensando en las nuevas tecnologías, usted ve... \\
\hline Más amenazas que oportunidades & $35,7 \%$ \\
\hline Más oportunidades que amenazas & $61,1 \%$ \\
\hline No Sabe - No Responde & $3,2 \%$ \\
\hline
\end{tabular}

Fuente: PNUD 2006: 234

Respecto al saber científico, de acuerdo a lo expresado por el informe del PNUD del año 2004 (ver cuadro 3), la mayoría de la población chilena considera que quienes deben tomar las decisiones respecto al devenir de Chile, idealmente serían aquellas personas que cuentan con conocimientos técnicos y científicos para hacerlo, esto incluso por sobre aquellos que han sido elegidos por la mayoría. 
Esta idea sugiere que quien no domine el lenguaje tecnológico y científico actual, corre el riesgo de quedar excluido de la sociedad, el desarrollo tecnológico y científico ha transformado la dinámica inclusión-exclusión social que antes estuvo determinada por la acumulación de capital.

\section{Cuadro 3.}

\begin{tabular}{|c|c|}
\hline $\begin{array}{l}\text { En todos los países hay personas que toman las decisior } \\
\text { siguen. Según usted, ¿quiénes son los que, principalmer } \\
\text { las decisiones? }\end{array}$ & \\
\hline Los que por tradición siempre lo han hecho & $5,1 \%$ \\
\hline Los que tienen los conocimientos técnicos para hacerlo & $43,2 \%$ \\
\hline Los que la mayoría ha elegido & $20,5 \%$ \\
\hline Los que pueden crear riqueza y dar trabajo & $21,4 \%$ \\
\hline Los que la ley designa para hacerlo & $8,7 \%$ \\
\hline No sabe - No Responde & $1,1 \%$ \\
\hline
\end{tabular}

Fuente: PNUD 2004: 285

Finalmente, la sensación general de la ciudadanía en la actualidad es que la modernización ha favorecido el desarrollo del país en determinados tópicos. Tal hecho se expresa en el informe del PNUD de 2004, donde "en general se percibe que el actual escenario del país, facilita la emergencia de oportunidades en el ámbito educativo, en el acceso de bienes materiales y en facilitar la individualización" (PNUD 2004: 289). La ciudadanía chilena, por tanto, está inmersa en este proceso y no manifiesta un aparente estado de disconformidad, sin embargo, esto no reflejaría otra cosa más que el correcto desempeño de los sistemas abstractos, incluso de la clase política, para legitimarse por sobre las incertidumbres.

Los sistemas abstractos se han transformado a la vez en poderes fácticos, tanto el Estado, la clase política, la generación de nuevas tecnologías, el saber científico, la proliferación de los medios de comunicación, etcétera. Todos obedecen a intereses particulares.

\section{Congestión y malestares de la modernización}

Según Beck (1998), la congestión es una parálisis general en la modernización causada por un debilitamiento del proceso de implementación de la industrialización. El autor dice que esta parálisis generalizada viene acompañada a la subpolitización; los modernizadores, al igual que sus críticos, quedan atrapados en la rigidez de los puntos de vista e intereses fomentados. Este debilitamiento del proceso de imple- 
mentación de la industrialización, que antes estaba tan bien lubricado por el consenso, impide el proceso y anuncia la auto limitación y el autocontrol anárquico de un proceso de industrialización que, anteriormente, carecía de frenos (Beck 1998). Esta congestión crea quiebres o recaídas de la confianza en los sistemas abstractos. En el nivel de la vida cotidiana, la retirada de la confianza puede tener diversas formas, algunas de las cuales son enteramente marginales a la persistencia de los propios sistemas abstractos. "No importa demasiado que unas pocas personas decidan marginarse de forma mas o menos completa de los sistemas abstractos que las rodean, por ejemplo, como el grupo religioso los Testigos de Jehová. Pero algunos quiebres o recaídas de la confianza tienen, no obstante, implicaciones mucho más amplias. Una progresiva aceleración de la desconfianza en un banco, o en un gobierno, puede conducir a su colapso" (Robles 2000).

La congestión social es entendida también por Giddens (1997) como la fase posterior al proceso de compulsión social. Se caracteriza por la pérdida de la confianza por parte de la ciudadanía hacia los sistemas abstractos y hacia el orden establecido en el proceso de modernización, ya que estos últimos no pueden cumplir con las promesas que justificaban su existencia. La individualización lleva a un estado de inconsciencia que transforma al hombre en un ser cuyas acciones son en gran parte repetitivas e inconscientes, esto producto de la confianza al proyecto de modernidad y a la dinámica de la modernización. Sin embargo, en un determinado instante este proceso se estanca y el hombre sale de esta perplejidad estructural en la que está inmerso y asume que las certezas que guiaron su vida ya no son tales. Esto lleva a la emergencia de la subpolitización de la ciudadanía, que en bloque amenaza el orden establecido en conjunto por la política y los sistemas abstractos y que no pueden mantener, generando congestión social, desafiliación estructural y finalmente llevando a la sociedad a un proceso que Beck y Giddens definen como modernización reflexiva (1997).

Es en este sentido que Beck (1997) habla de una "subpolítica", donde los nuevos movimientos, grupos y ONGs comienzan a ser verdaderos protagonistas en la escena mundial, esta escena, definida por Giddens (1999) como "la tercera vía"1, marcada por las grandes revo-

1 El autor propone la renovación de la socialdemocracia en un mundo en el que las ideas de la vieja izquierda han quedado obsoletas, mientras que las de la nueva derecha son inadecuadas y contradictorias. 
luciones de nuestro tiempo: la globalización, las transformaciones de la vida personal y la relación del hombre con la naturaleza. Para ello, el tema de la modernidad reflexiva es condición fundamental para la nueva política que busca establecer una nueva relación entre individuo y comunidad, una redefinición de derechos y obligaciones.

Al respecto se puede apreciar, que este fenómeno de subpolitización entendido en los términos que Beck y Giddens lo definen, en ningún caso se viviría a plenitud en nuestro país (1997).

Por ejemplo, para Garretón (2005) la organización social se da en función de temáticas puntuales que involucran a grupos específicos, cuyas metas no implican necesariamente la transformación de la sociedad. Agrega que pese al creciente fenómeno de deslegitimación de la clase política tampoco la sociedad civil se articula masivamente para crear un nuevo referente: "Solo hay elementos específicos y dispersos de ese escenario (subpolitización) que sí se identifican, es decir, uno los puede ver... pero que difícilmente en su globalidad integrada, es decir, a la sociedad civil en su mayoría integrada, se pueda presentar" (Garretón 2005).

Estas demandas particulares que afloran de grupos específicos, tampoco están articuladas entre sí, por lo que difícilmente surgirá un gran movimiento social marcado por el desencanto al estilo de vida predominante. La subpolitización en Chile se caracteriza por la movilización social y el descontento social, y no por los movimientos sociales y el desencanto social.

En el informe del PNUD de 1998 se planteó que ya en la década de los noventa, el proceso de modernización generó malestares, sin embargo, estas molestias no llevaban a la sociedad a la protesta y a la manifestación pública, sino más bien, a una sensación interna de frustración y de complacencia, tal cual sucede en el presente. Por otra parte, este informe de 1998 planteó que entonces existió una creciente desconfianza a los sistemas funcionales por parte de la ciudadanía, principalmente al sistema de salud, a la previsión social, al Estado y en general a lo que hemos denominado sistemas abstractos. Sin embargo, muchas de estas incertidumbres parecieran ser que se han ido dispersando en el tiempo, ya que los informes del 2004 y 2006 reflejan una aparente confianza en el escenario de la modernización actual y en sus sistemas abstractos, lo que explica lo difícil que resultaría la consolidación de una subpolitización activa que amenace con fuerza la dinámica actual del ordenamiento social del país. 
Al hablar de congestión social, hablamos de una sociedad donde los malestares creados por la modernización y los riesgos que estos generan, superan el control y las respuestas que puedan tener las estructuras formales y los sistemas abstractos. Si nos remitimos a esto, podemos decir que en Chile no se produciría una congestión social. Más bien Chile viviría lo que a juicio de Beck (1997) y Luhmann (1998) se denominaría una "Sociedad del Riesgo Residual", donde la ciudadanía es consciente de los riesgos que surgen de la modernización, pero dada su confianza en la controlabilidad que tendrán los sistemas abstractos a estas incertidumbres no se transformará en subpolitización.

Esta característica pasiva y residual de la ciudadanía se puede apreciar en el informe del PNUD del año 2004, donde se expresa que la mayoría de los chilenos, no se siente dispuesto a organizar socialmente a la comunidad para protestar o manifestarse ante alguna demanda, y tampoco vemos que los mecanismos tradicionales de expresión del malestar social, tengan cabida y representatividad en la comunidad (ver tablas 4 y 5 ).

Cuadro 3.

\begin{tabular}{|l|c|c|c|}
\hline \multicolumn{2}{|l}{ ¿Estaría usted dispuesto a hacerse cargo de...? } \\
\hline $\begin{array}{l}\text { Organizar a algún grupo de personas para hacer un } \\
\text { reclamo a una autoridad pública o empresa. }\end{array}$ & $33,6 \%$ & $64,9 \%$ & $1,5 \%$ \\
\hline $\begin{array}{l}\text { Organizar alguna protesta (paro, manifestación) con- } \\
\text { tra una autoridad pública o empresa. }\end{array}$ & $17,9 \%$ & $79,4 \%$ & $2,7 \%$ \\
\hline $\begin{array}{l}\text { Organizar o coordinar la realización de alguna activi- } \\
\text { dad pública (actividad solidaria, campaña de utilidad } \\
\text { colectiva) }\end{array}$ & $47,0 \%$ & $50,0 \%$ & $3 \%$ \\
\hline
\end{tabular}

Fuente: PNUD 2004: 293

Cuadro 4.

\begin{tabular}{|l|c|}
\hline \multicolumn{2}{|l|}{$\begin{array}{l}\text { De los siguientes, ¿Cuál cree que es el medio más útil para que las personas } \\
\text { como usted puedan reclamar sus derechos? }\end{array}$} \\
\hline Organizando una protesta & $7,6 \%$ \\
\hline A través de una denuncia en tribunales & $22,5 \%$ \\
\hline Reclamando en los servicios públicos & $26,7 \%$ \\
\hline A través de la radio, diarios o televisión & $33,9 \%$ \\
\hline A través de internet & $2,4 \%$ \\
\hline Ninguno & $5,3 \%$ \\
\hline NS-NR & $1,6 \%$ \\
\hline
\end{tabular}

Fuente: PNUD 2006: 231 
Respecto al futuro, las cifras de los informes de Desarrollo Humano, desde el año 1998 hasta el 2006, más reflexiones sociopolíticas como las de Garretón (2000) y Robles (2000), nos muestran que es complicado creer que el fenómeno de subpolitización podría ir aumentando en el país.

Finalmente, y según lo descrito en el PNUD 2006, en el futuro la ciudadanía chilena acrecentará su confianza en el sistema económico, el cual cree que le brindará mayores oportunidades de desarrollo personal. (Ver cuadro 5).

Cuadro5.

\begin{tabular}{|l|c|}
\hline ¿Cómo cree que será la situación económica de su familia en cinco años más? \\
\hline Mejor que la actual & $56,0 \%$ \\
\hline Igual & $32,2 \%$ \\
\hline Peor que la actual & $8,0 \%$ \\
\hline NS-NR & $3,8 \%$ \\
\hline
\end{tabular}

Fuente: PNUD 2006: 248

Respecto al desarrollo científico y tecnológico sí surgen algunas incertidumbres sobre el efecto que tendrá en el ámbito laboral, la proliferación de las nuevas tecnologías y también respecto al problema del acceso a ellas, ya que quienes posean más de las nuevas tecnologías concentrarán más poder (ver cuadro 6).

\section{Cuadro 6.}

\begin{tabular}{|l|c|}
\hline $\begin{array}{l}\text { Si usted piensa en el futuro de la sociedad chilena, ¿qué es más probable } \\
\text { que ocurra? }\end{array}$ \\
\hline $\begin{array}{l}\text { Con las nuevas tecnologías la gente común y corriente tendrá más } \\
\text { poder. }\end{array}$ & $1,8 \%$ \\
\hline $\begin{array}{l}\text { Con las nuevas tecnologías el poder lo concentrarán los científicos y } \\
\text { los técnicos. }\end{array}$ & $50,5 \%$ \\
\hline No Sabe - No Responde & $7,7 \%$ \\
\hline
\end{tabular}

Fuente: PNUD 2006: 234

Pero por otro lado, las incertidumbres anteriores chocan con una sensación general de que el camino hacia el desarrollo tecnológico es el correcto y es el que debe guiar el destino del país.

En este sentido se plantea que con esta lógica, la ciudadanía considera que Chile, en un futuro, sería un país desarrollado, pero a la vez nos muestra la realidad de un país que aparentemente posee más certezas que incertidumbres respecto a la modernización, y que por 
lo tanto hasta este instante no vive una subpolitización creciente ni congestión social, ni tampoco Modernización Reflexiva.

\section{Conclusiones}

El fenómeno de la modernización en Chile es un campo abierto, propicio para la investigación y la reflexión en el mundo académico, político, científico y social.

A la hora de analizar los cambios y transformaciones de nuestra sociedad, consideramos que los rasgos compulsivos que se hacen presentes en nuestra sociedad son similares a los que menciona Giddens (1997)para definir a las sociedades compulsivas de occidente.

El vínculo entre la sociedad civil y los sistemas abstractos está sustentado en la confianza, dado que la ciudadanía valida el saber tecnológico y científico por sobre incluso algunos elementos de la democracia. A la vez espera gozar de las nuevas tecnologías que vayan surgiendo y cree que estas mismas, facilitarán su vida en el futuro. Por lo que en la actualidad se redefine la dinámica inclusión o exclusión social, antes caracterizada por la acumulación o no de capital, pero hoy definida en función del acceso y manejo de las nuevas tecnologías y del saber científico.

La sociedad chilena es una sociedad compulsiva, donde su gran masa de habitantes, sigue a la par un proyecto común sin mayor concientización ni consideración respecto de las consecuencias que traerá este modelo de vida.

Finalmente, luego de este análisis podemos plantear que nuestra sociedad, está más identificada con el escenario compulsivo que a un estado de congestión social y de creciente subpolitización, particularmente por lo que ya se ha descrito anteriormente sobre el vínculo entre la ciudadanía y los sistemas abstractos, pero también, porque si bien en nuestro país sí existen riesgos sociales inesperados producto de una mala ejecución de alguna acción propia de la modernización, estos no generan subpolitización en la ciudadanía. Sólo se dan algunos rasgos de organización social, más vinculados conceptualmente a movilizaciones sociales focalizadas, que a un movimiento social masivo de desencanto global. 
Podemos decir por último que en nuestra sociedad actual, no sólo existe una confianza sobre las bases centrales de la modernización, si no también existe una percepción mayoritaria de que en el futuro, las cosas "seguirán estando bien" o irán mejorando, pero pareciera que siempre en el marco de la modernización.

Respecto al sentido base de esta reflexión, podemos plantear que en nuestra sociedad, los aspectos distintivos que caracterizan una sociedad reflexiva se hacen presentes sólo parcialmente.

Consideramos que la Compulsividad es el escenario que más representa a la sociedad chilena, esto debido a que gran parte de las secciones individuales obedecen a un orden global legitimado por las estructuras abstractas, y que no genera mayor conflictividad en la sociedad civil. Las acciones repetitivas e inconscientes también representan otro rasgo distintivo. Podemos apreciar la compulsividad particularmente en la manera de enfrentar el consumismo y la confianza ciega en las nuevas tecnologías y en los medios de comunicación.

A diferencia de lo que plantearía Giddens y Beck (1997), en Chile la fase de compulsividad no conduciría del todo a un proceso de cambio social reflexivo por parte de la sociedad, producto del arraigo e identificación que tiene nuestra sociedad con la dinámica actual de modernización.

Respecto de la congestión y los malestares de la modernización, vemos que la sociedad chilena se caracterizaría más por vivir riesgos residuales, que riesgos que deriven en incertidumbre general, tal como podemos apreciar con el fenómeno del Transantiago, que a pesar de vivir un año de constantes problemas e irregularidades, no ha sido motivo de un conflicto social estandarizado, producto de lo que hemos denominado "confianza en los sistemas abstractos", es decir, en nuestra sociedad la gente percibe que hay riesgos que generan malestares, pero que no llevarán a una transformación social, dado que predomina la sensación de controlabilidad de los riesgos por parte de los sistemas abstractos. Chile por tanto no vive un proceso de subpolitización, y por ende el traspaso desde la sociedad compulsiva a la congestión social aún no se da.

"Somos una sociedad con pocos vínculos comunitarios. Si te va mal en el mercado, te quedas prácticamente desnudo. Los fracasos son vividos como culpas y 
no como fallas del sistema. Por lo tanto, los fracasos conducen al sicólogo y no a una rebelión en la calle".

(Tironi 2002) ${ }^{2}$

\section{Referencias bibliográficas}

BECK, U. (1998).

(2000).

GARRETÓN, M. (2000).

GIDDENS, A. (1993).
La Sociedad del Riesgo. Hacia una nueva modernidad. Barcelona: Paidós.

Las consecuencias perversas de la Modernidad: Modernidad, contingencia y riesgo. Madrid: Alianza.

La sociedad en que vivi(re)mos. Introducción sociológica al cambio de siglo. Santiago, Chile: Ediciones LOM.

¿Cómo pensar a Chile en el Bicentenario. Conferencia en palacio de la moneda, 30 de Marzo del 2005, Chile.

Consecuencias de la modernidad. Madrid: Alianza,

"Vivir en una Sociedad Postradicional". Alianza, Madrid.

GIDDENS, A., BECK, LASH. (1997).

Modernización Reflexiva: Política, tradición y estética en el orden moderno. Madrid: Alianza.

PROGRAMA DE LAS NACIONES UNIDAS PARA EL DESARROLLO (1998).

Informe de Desarrollo Humano. Las paradojas de la Modernización. Santiago de Chile: AUTOR.

(2000). Informe de Desarrollo Humano. Más sociedad para gobernar el futuro. Santiago de Chile: AUTOR.

(2002). Informe de Desarrollo Humano. Nosotros los chilenos: Un desafío cultural. Santiago de Chile: AUTOR.

(2004). Informe de Desarrollo Humano. El poder: ¿Para qué y para quién? Santiago de Chile: AUTOR.

(2006). Informe de Desarrollo Humano. Las nuevas tecnologías: ¿un salto al futuro? Santiago de Chile: AUTOR.

LUHMANN, N. (1998). O Observaciones de la modernidad. Barcelona: Paidós.

2 Informe de Desarrollo Humano. "Nosotros los chilenos: Un desafío cultural". Programa de las Naciones Unidas para el Desarrollo. 2002, Santiago de Chile. 
QUIJANO, A. (1989). Modernidad, Identidad y Utopía en América Latina. Lima: Ediciones Sociedad y Política.

ROBLES, F. (2005). Contramodernidades y Globalizaciones Paradójicas: La configuración de las sociedades periféricas de riesgo. Esbozos para reubicar lo político. Revista MAD, 12. http://www.sociales. uchile.cl/publicaciones/mad/12/paper02.pdf.

(2000). El desaliento inesperado de la modernidad. Molestias, irritaciones y oportunidades de la sociedad del riesgo. Concepción: Ediciones Universidad de Concepción. 